Tropical Journal of Pharmaceutical Research June 2020; 19 (6): 1197-1201

ISSN: $1596-5996$ (print); 1596-9827 (electronic) (C) Pharmacotherapy Group, Faculty of Pharmacy, University of Benin, Benin City, 300001 Nigeria

\title{
Hesperetin protects SH-SY5Y cells against 6- hydroxydopamine-induced neurotoxicity via activation of NRF2/ARE signaling pathways
}

\author{
Jing Li ${ }^{1}$, Yue Liu', Li Wang ${ }^{2}$, Zhaowei Gu${ }^{1}$, Zhigang Huan², Hui Fu², Qishuai \\ Liu $^{2 *}$ \\ ${ }^{1}$ Encephalopathy Center, The 3rd Affiliated Hospital of Changchun University of Chinese Medicine, Changchun City, Jilin \\ Province 130000, ${ }^{2}$ Department of Nephrology, The First Hospital of ZiBo, Zibo City, Shandong Province 255200, China
}

*For correspondence: Email: QishuaiLiufjk@163.com; Tel: +86-5334251458

Sent for review: 10 March 2020

Revised accepted: 28 May 2020

\begin{abstract}
Purpose: To investigation the protective effects of hesperetin against 6-hydroxydopamine (6-OHDA)induced neurotoxicity.

Methods: SH-SY5Y cells were incubated with 6-OHDA to create an in vitro model of neurotoxicity. This model was used to test the neuroprotective effects of hesperetin. Cell viability was assessed by MTT and lactate dehydrogenase $(L D H)$ release assays. Flow cytometry and western blot were used to quantify apoptosis. Oxidative stress was evaluated by determining intracellular glutathione (GSH), malondialdehyde (MDA), superoxide dismutase (SOD), and reactive oxygen species (ROS).

Results: In SH-SY5Y cells, treatment with 6-OHDA decreased cell viability and promoted $\mathrm{LDH}$ release. However, exogenous hesperetin protected against 6-OHDA-mediated toxicity. Similarly, although incubation with 6-OHDA induced apoptosis and increased cleaved caspase-3 and -9 levels, treatment with hesperetin protected against these effects. Treatment with 6-OHDA also led to significant oxidative stress, as indicated by reduced GSH and SOD levels and increased MDA and ROS levels in SH-SY5Y cells. However, these changes were reversed by pre-treatment with hesperetin. Of interest, hesperetin led to changes in 6-OHDA-induced expression of NRF2, heme oxygenase-1 (HO-1), glutamate-cysteine ligase (GCL) catalytic subunit (GCLC), and GCL modulatory (GCLM).

Conclusion: Hesperetin protects against cell toxicity, apoptosis, and oxidative stress via activation of NRF2 pathway in a 6-OHDA-induced model of neurotoxicity. Future studies should investigate the use of hesperetin as a potential therapeutic approach for prevention or management of Parkinson's disease.
\end{abstract}

Keywords: Hesperetin, 6-OHDA, Neurotoxicity, NRF2, Parkinson's disease

\begin{abstract}
This is an Open Access article that uses a fund-ing model which does not charge readers or their institutions for access and distributed under the terms of the Creative Commons Attribution License (http://creativecommons.org/licenses/by/4.0) and the Budapest Open Access Initiative (http://www.budapestopenaccessinitiative.org/read), which permit unrestricted use, distribution, and reproduction in any medium, provided the original work is properly credited.
\end{abstract}

Tropical Journal of Pharmaceutical Research is indexed by Science Citation Index (SciSearch), Scopus, International Pharmaceutical Abstract, Chemical Abstracts, Embase, Index Copernicus, EBSCO, African Index Medicus, JournalSeek, Journal Citation Reports/Science Edition, Directory of Open Access Journals (DOAJ), African Journal Online, Bioline International, Open-J-Gate and Pharmacy Abstracts

\section{INTRODUCTION}

Parkinson's disease (PKD) is a nerve degenerative disease without effective neuroprotective treatment [1]. However, there is currently no treatment for PKD. Improper balance between the intracellular antioxidant and oxidation systems contributes to the development of PKD [2]. Of note, NRF2 protects against oxidative neurological stress [3] and 
plays a central role in the biology of PKD. NRF2mediated transcriptional deletion increases the susceptibility of dopaminergic neurons to oxidative stress during PKD [4], and mice that are deficient in NRF2 are more susceptible to toxin-mediated damage of neurons in the substantia nigra than wild-type mice [5]. Moreover, activation of NRF2 leads to neuroprotection against toxins [6,7]. Therefore, NRF2 seems to be a potentially valuable target for new therapies aimed to treat or prevent PKD.

Hesperidin is a common, inexpensive plant flavonoid that is derived from citrus plants, such as sweet oranges and lemons [8]. Hesperidin has many pharmacological properties, including anti-hypercholesterolemia, anti-inflammatory, antioxidant, and neuroprotective effects [8]. Hesperetin is an aglycone of hesperidin that is produced by the intestinal microflora and has been shown to have anticancer effects in a mouse model of prostate cancer [9]. Recently, hesperidin was reported to prevent $\mathrm{H}_{2} \mathrm{O}_{2}$-induced oxidative damage by upregulating the NRF2 pathway [10]. Important to the topic of this work, hesperetin has been shown to suppress oxidative stress and protect against Alzheimer's disease [11]. However, the potential benefits and mechanisms of hesperetin in the treatment of PKD remain poorly understood to date.

In this study, we established a 6-OHDA-induced cell model of PKD to evaluate the therapeutic potential of hesperetin and the role of the NRF2 signaling pathway in PKD.

\section{EXPERIMENTAL}

\section{Cells and treatment}

Cells were cultured in DMEM-F12 medium (Lonza, Basel, Switzerland) with $10 \%$ FBS (Gibco, Gaithersburg, MD, USA) in a constant temperature incubator. Cells were treated with 10,20 , or $50 \mu \mathrm{M}$ hesperetin for $2 \mathrm{~h}$ and then treated with $100 \mu \mathrm{M} 6-\mathrm{OHDA}$ for $24 \mathrm{~h}$.

\section{Evaluation of cell viability}

Cells $\left(1 \times 10^{3}\right.$ per well) were incubated with 5 $\mathrm{mg} / \mathrm{mL}$ of MTT for $4 \mathrm{~h}$. After removing the media, cells were incubated and the absorbance was read at $490 \mathrm{~nm}$.

\section{Assessment of lactate dehydrogenase (LDH) release}

After incubation with hesperetin and 6-OHDA, $100 \mu \mathrm{L}$ of cell culture medium was incubated with
$\mathrm{LDH}$ reaction reagent for measurement of $\mathrm{LDH}$ release.

\section{Flow cytometry}

Cells were harvested and resuspended in binding buffer with R-phycoerythrin (PE; $5 \mu \mathrm{L}$, $100 \mu \mathrm{g} / \mathrm{mL}$ ) and ribonuclease $(1 \mathrm{U} / \mathrm{mL})$. The cells were incubated with $5 \mu \mathrm{L}$ of FITC-conjugated Annexin $V$ before analyzed by FACS flow cytometry (Attune; Life Technologies, Darmstadt, Germany).

\section{Measurement of oxidative stress}

After treatment with 10,20 , or $50 \mu \mathrm{M}$ hesperetin for $2 \mathrm{~h}$ and incubation with $100 \mu \mathrm{M}$ 6-OHDA for $24 \mathrm{~h}$, cells were harvested, and levels of MDA, SOD, GSH, and ROS were determined using commercially available kits (Beyotime Biotechnology, Shanghai, China).

\section{Western blot assay}

Cell proteins were extracted and total protein concentrations were determined via BCA assay. Proteins were separated by SDS-PAGE and then transferred onto a PVDF membrane, which was then incubated with primary antibodies against cleaved caspase-3, cleaved caspase-9, NRF2, $\mathrm{HO}-1, \mathrm{GCLM}, \mathrm{GCLC}$, and GAPDH (Abcam, Cambridge, MA, USA) followed by a secondary antibody. Relative densitometries were analyzed using Image $\mathrm{J}$ software and compared to GAPDH as a loading control.

\section{Statistical analysis}

The results were shown as mean \pm SD. Graph Pad Prism 5 was used to conduct one-way analysis of variance. Comparisons resulting in $p$ $<0.05$ were considered statistically significant.

\section{RESULTS}

\section{Hesperetin protected against 6-OHDA- mediated decrease in cell viability}

As expected, treatment of SH-SY5Y cells with 6OHDA $(100 \mu \mathrm{M})$ decreased cell viability and promoted $\mathrm{LDH}$ release relative to healthy control cells. However, pre-treatment with hesperetin reversed these toxic effects in a dose-dependent manner (Figure 1).

\section{Hesperetin protected against 6-OHDA- mediated apoptosis}

Treatment with 6-OHDA led to increased apoptosis relative to control cells (Figure $2 \mathrm{~A}$ ). 
However, pre-treatment with hesperetin reversed this effect (Figure 2 A). Expression of several proteins involved in apoptosis, such as cleaved caspase-3 and -9 , was increased after incubation with 6-OHDA, but this effect was attenuated in cells pre-treated with hesperetin (Figure $2 \mathrm{~B}$ ).

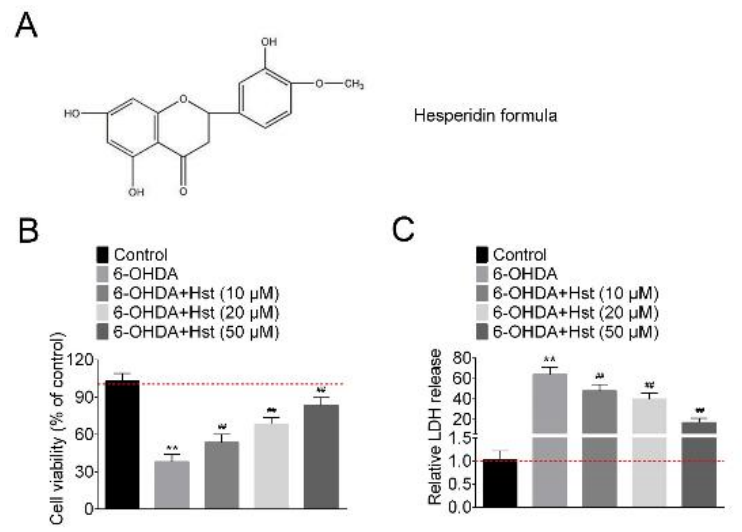

Figure 1: Hesperetin reversed the effects of 6-OHDA on cell viability. (A) Chemical structure of hesperetin (B) Cell viability was measured by MTT assay. (C) $\mathrm{LDH}$ release was measured; ${ }^{*} p<0.05 ;{ }^{* *},{ }^{\# \#} p<0.01$

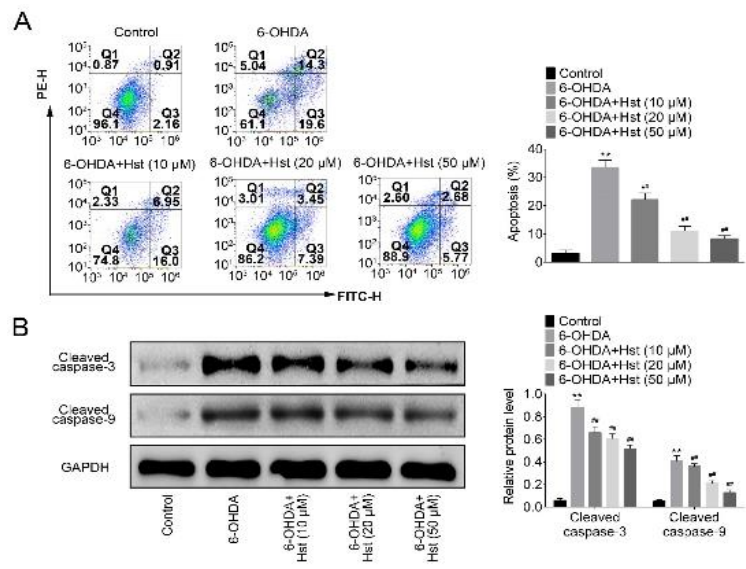

Figure 2: Hesperetin reduced apoptosis of cells treated with 6-OHDA. (A) Cellular apoptosis was detected by flow cytometry. (B) Protein expression was detected by western blots; ${ }^{* *}, \ldots<0.01$

\section{Hesperetin reversed the effects of 6-OHDA on oxidative stress}

The expression of antioxidant enzymes, including GSH and SOD, was decreased by 6-OHDA treatment. However, hesperetin reversed these results (Figure $3 A$ and $B$ ). Similarly, the levels of MDA and ROS were increased after treatment with 6-OHDA but were relatively suppressed after pre-treatment with hesperetin (Figure $3 \mathrm{C}$ and D).

\section{Hesperetin reversed the effects of 6-OHDA on NRF2 pathway}

Treatment with 6-OHDA decreased the expression of NRF2, HO-1, GCLC, and GCLM compared to control cells (Figure 4). However, pre-treatment with hesperetin caused over activation of the NRF2 pathway, as seen by relative increases in expression of NRF2, HO-1, GCLC, and GCLM (Figure 4).
A

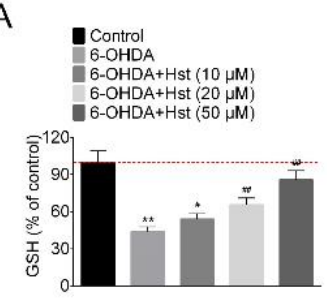

C

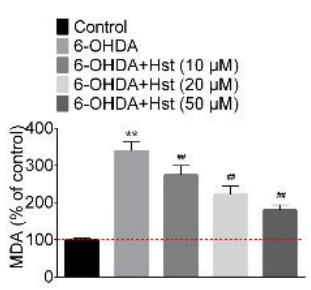

B

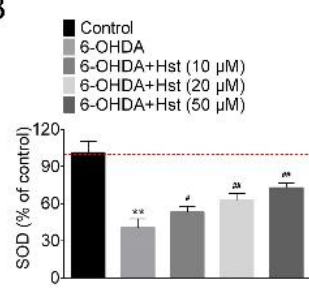

D

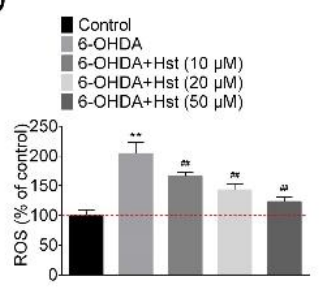

Figure 3: Hesperetin reversed the effects of 6-OHDA on GSH (A), SOD(B), MDA(C) and ROS(D) level. ${ }^{\#} p<$ $0.05 ;{ }^{* *}, \#$ \# $p<0.01$
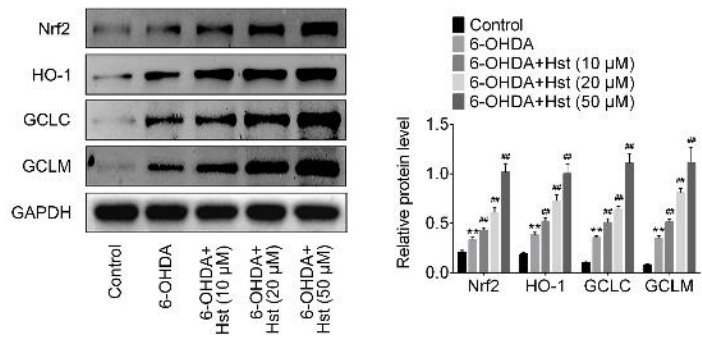

Figure 4: Hesperetin reversed the effects of 6-OHDA on the NRF2 pathway. Western blotting was used to measure protein expression. ${ }^{* *}, \ldots<0.01$

\section{DISCUSSION}

Flavonoids may play a useful role in the prevention and treatment of PKD due to their antioxidant activities [12]. Naringenin, a flavonoid found in grapefruit, has been shown to provide neuroprotection in PKD [13]. In this work, hesperetin, a flavonoid, had neuroprotective effects in a cell model of PKD through its antioxidant and anti-apoptotic effects.

Treatment with 6-OHDA promotes ROS accumulation and apoptosis of dopaminergic cells in rats [14] and is thus commonly used to 
create in vitro models of PKD [15]. Importantly, SH-SY5Y cells express tyrosine hydroxylase and mimic dopaminergic neurons [16]. Studies have shown that 6-OHDA promotes oxidative stress by uncoupling oxidative phosphorylation and inducing toxicity, similar to native pathological processes in PKD [17]. In this work, SH-SY5Y cells were treated with 6-OHDA to create an in vitro model of PKD. The death of dopamine neurons is one of the characteristics of PKD. Therapeutics that counteract the effects of neurotoxins, such as 6-OHDA, to protect neurons may prove valuable for the treatment of PKD [18].

This study showed that pre-treatment with hesperetin reversed 6-OHDA-mediated reductions in cell viability, consistent with a previous report showing that hesperetin protected SH-SY5Y cells against rotenoneinduced toxicity [19]. Necrosis and apoptosis are major forms of cell death during PKD [20]. A previous study indicated that $\mathrm{LDH}$ is released into the supernatant when necrosis occurs [18]. However, another report revealed that preadministration of hesperetin decreased neuronal cell apoptosis [21]. Consistent with these prior studies, we found that treatment with hesperetin demonstrated protective effects against 6-OHDA toxicity through inhibition of $\mathrm{LDH}$ release and prevention of apoptosis.

PKD has been suggested to be the result of free radical-induced oxidative stress [22]. Under normal conditions, various antioxidant enzymes, including GSH and SOD, detoxify the cell by removing free radicals [23]. The accumulation of MDA and ROS can upset the balance of antioxidant defense systems and result in oxidative stress [2]. Previous studies showed that hesperetin exerts a neuroprotective effect by activating the antioxidant enzyme system and inhibiting oxidative damage [24] and prevents ROS accumulation in a rotenone-induced $\mathrm{SH}$ SY5Y cell model [19]. Consistently, this study found that hesperetin had antioxidant effects in cells treated with 6-OHDA treatment, primarily through increasing the expression of antioxidant enzymes (GSH and SOD) and reducing the relative concentration of markers of oxidative stress (MDA and ROS).

Several signaling pathways play important roles in the antioxidant response to PKD [25]. NRF2 is a key regulator of cytoprotective genes, such as HO-1, GCLC, and GCLM, and plays a central role in managing oxidative stress during PKD [13]. Activation of the NRF2 pathway, as evidenced by the increased expression of NRF2, HO-1, GCLC, and GCLM, leads to neuroprotection of SH-SY5Y cells treated with 6OHDA [13]. A previous study showed that hesperetin exerts neuroprotective effects by activating the NRF2 pathway [26]. Similarly, we found that hesperetin upregulated NRF2, HO-1, GCLC, and GCLM, thus activating the NRF2 pathway and reducing oxidative stress. However, further investigations in animal models of disease will be required to more completely reveal the neuroprotective role of hesperetin in the context of PKD.

\section{CONCLUSION}

Hesperetin protects against apoptosis and oxidative stress in a 6-OHDA-induced SH-SY5Y cell model of PKD. These antioxidant effects were mediated through activation of the NRF2 pathway. These findings indicate that hesperetin may have a neuroprotective role in the management of PKD.

\section{DECLARATIONS}

\section{Conflict of interest}

No conflict of interest is associated with this work.

\section{Contribution of authors}

We declare that this work was done by the authors named in this article and all liabilities pertaining to claims relating to the content of this article will be borne by the authors.

\section{Open Access}

This is an Open Access article that uses a funding model which does not charge readers or their institutions for access and distributed under the terms of the Creative Commons Attribution License (http://creativecommons.org/licenses/by/ 4.0) and the Budapest Open Access Initiative (http://www.budapestopenaccessinitiative.org/rea d), which permit unrestricted use, distribution, and reproduction in any medium, provided the original work is properly credited.

\section{REFERENCES}

1. Kim J-B, Kopalli SR, Koppula SJTJoPR. Cuminum cyminum Linn (Apiaceae) extract attenuates MPTPinduced oxidative stress and behavioral impairments in mouse model of Parkinson's disease. Trop J Pharm Res 2016; 15(4): 765-772.

2. Hwang $O$. Role of Oxidative Stress in Parkinson's Disease. Exp Neurobiol 2013; 22(1): 11-17. 
3. Johnson JA, Johnson $D A$, Lee J-M, Li J, Kraft $A D$, Calkins $M J$, Jakel RJ. The Nrf2-ARE pathway: A potential therapeutic target for neurodegenerative diseases. Int Congress Series 2007; 1302: 143-153.

4. Burton NC, Kensler TW, Guilarte TR. In vivo modulation of the Parkinsonian phenotype by Nrf2. Neurotoxicology 2006; 27(6): 1094-1100.

5. Jazwa A, Rojo Al, Innamorato NG, Hesse M, FernandezRuiz J, Cuadrado A. Pharmacological targeting of the transcription factor Nrf2 at the basal ganglia provides disease modifying therapy for experimental parkinsonism. Antioxid Redox Sign 2011; 14(12): 23472360.

6. Chen $P C$, Vargas MR, Pani AK, Smeyne RJ, Johnson $D A$, Kan YW, Johnson JA. Nrf2-mediated neuroprotection in the MPTP mouse model of Parkinson's disease: Critical role for the astrocyte. $P$ Natl Acad Sci USA 2009; 106(8): 2933-2938.

7. Jakel RJ, Townsend JA, Kraft AD, Johnson JA. Nrf2mediated protection against 6-hydroxydopamine. Brain Res 2007; 1144: 192-201.

8. Cho J. Antioxidant and neuroprotective effects of hesperidin and its aglycone hesperetin. Arch Pharm Res 2006; 29(8): 699-706.

9. Shirzad M, Beshkar P, Heidarian E. The effects of hesperetin on apoptosis induction and inhibition of cell proliferation in the prostate cancer PC3 cells. J HerbMed Pharm 2015; 4: 121-124.

10. Zhu C, Dong Y, Liu H, Ren H, Cui Z. Hesperetin protects against H2O2-triggered oxidative damage via upregulation of the Keap1-Nrf2/HO-1 signal pathway in ARPE-19 cells. Biomed Pharmacother 2017; 88: 124133.

11. Kheradmand E, Hajizadeh Moghaddam A, Zare $M$. Neuroprotective effect of hesperetin and nanohesperetin on recognition memory impairment and the elevated oxygen stress in rat model of Alzheimer's disease. Biomed Pharmacother 2018; 97: 1096-1101.

12. Magalingam $K B$, Radhakrishnan $A K$, Haleagrahara $N$. Protective Mechanisms of Flavonoids in Parkinson's Disease. Oxid Med Cell Longev 2015; 2015: 314560.

13. Lou $H$, Jing $X$, Wei $X$, Shi $H$, Ren $D$, Zhang $X$. Naringenin protects against 6-OHDA-induced neurotoxicity via activation of the Nrf2/ARE signaling pathway. Neuropharmacology 2014; 79: 380-388.

14. Wang YH, Yu HT, Pu XP, Du GH. Baicalein prevents 6hydroxydopamine-induced mitochondrial dysfunction in SH-SY5Y cells via inhibition of mitochondrial oxidation and up-regulation of DJ-1 protein expression. Molecules 2013; 18(12): 14726-14738.

15. Torres EM, Dunnett SB: 6-OHDA Lesion Models of Parkinson's Disease in the Rat. In: Animal Models of
Movement Disorders: Volume I. Edited by Lane EL, Dunnett SB. Totowa, NJ: Humana Press 2012; 267-279.

16. Takahashi T, Deng $Y$, Maruyama $W$, Dostert $P$, Kawai $M$, Naoi $M$. Uptake of a neurotoxin-candidate, (R)-1,2dimethyl-6,7-dihydroxy-1,2,3,4-tetrahydroisoquinoline into human dopaminergic neuroblastoma SH-SY5Y cells by dopamine transport system. J Neural Transm-Supp 1994; 98(2): 107-118.

17. Elkon H, Melamed E, Offen D. 6-Hydroxydopamine increases ubiquitin-conjugates and protein degradation: implications for the pathogenesis of Parkinson's disease. Cell Mol Neurobiol 2001; 21(6): 771-781.

18. Pan Z, Niu $Y$, Liang $Y$, Zhang $X$, Dong $M$. betaEcdysterone Protects SH-SY5Y Cells Against 6Hydroxydopamine-Induced Apoptosis via MitochondriaDependent Mechanism: Involvement of p38(MAPK)-p53 Signaling Pathway. Neurotox Res 2016; 30(3): 453-466.

19. Pedro ND, Cautain B, Cantizani J, Rodriguez L, Vicente $F$, RatnaTualsi, Koyyalamud SR. In vitro Neuroprotective Effects of Seven Natural Products Against Rotenone-induced Toxicity in a SH-SY5Y Neuroblastoma Cells Model for Parkinson's Disease. Pharmacologia 2016; 7(8): 361-370.

20. Davidovich $P$, Kearney CJ, Martin SJ. Inflammatory outcomes of apoptosis, necrosis and necroptosis. Biol Chem 2014; 395(10): 1163-1171.

21. Shagirtha K, Bashir N, MiltonPrabu S. Neuroprotective efficacy of hesperetin against cadmium induced oxidative stress in the brain of rats. Toxicol Ind Health 2017; 33(5): 454-468.

22. Kumar H, Lim H-W, More S, Kim B, Koppula S, Kim I, Choi D-K. The Role of Free Radicals in the Aging Brain and Parkinson's Disease: Convergence and Parallelism. Int J Mol Sci 2012; 13: 10478-10504.

23. Magalingam KB, Radhakrishnan $A$, Haleagrahara $N$. Rutin, a bioflavonoid antioxidant protects rat pheochromocytoma (PC-12) cells against 6hydroxydopamine (6-OHDA)-induced neurotoxicity. Int J Mol Sci 2013; 32(1): 235-240.

24. Choi EJ, Ahn WS. Neuroprotective effects of chronic hesperetin administration in mice. Arch Pharm Res 2008; 31(11): 1457-1462.

25. Fujita KA, Ostaszewski M, Matsuoka Y, Ghosh S, Glaab E, Trefois C, Crespo I, Perumal TM, Jurkowski W, Antony PM et al. Integrating pathways of Parkinson's disease in a molecular interaction map. Mol Neurobiol 2014; 49(1): 88-102.

26. Ikram M, Muhammad T, Rehman SU, Khan A, Jo MG, Ali $T$, Kim MO. Hesperetin Confers Neuroprotection by Regulating Nrf2/TLR4/NF-kappaB Signaling in an Abeta Mouse Model. Mol Neurobiol 2019; 56(9): 6293-6309. 